\title{
Qualidade de Vida e Grau de Independência Funcional em Pacientes com Acidente Vascular Cerebral
}

\author{
Quality of Life and Degree of Functional Independence in \\ Patients with Stroke
}

\author{
Michelle Morton Figueiredo Neves ${ }^{1}$, Laiz Helena de Castro Toledo \\ Guimarães $^{2}$
}

1.Acadêmica do Curso de Graduação em Fisioterapia do Centro Universitário de Lavras (UNILAVRAS). 2.Fisioterapeuta, Mestre, Docente e Coordenadora do Departamento de Fisioterapia do Centro Universitário de Lavras (UNILAVRAS).

\begin{abstract}
Resumo
Objetivo. Correlacionar a qualidade de vida (QV) com o grau de independência funcional de indivíduos que sofreram acidente vascular cerebral (AVC). Método. A amostra do presente estudo constituiu de 12 pacientes de ambos os gêneros, com média de idade de $60,58 \pm 10,34$ anos, não praticantes de exercício físico regular, que sofreram AVC de 6 meses a 9 anos e se encontravam em tratamento fisioterapêutico na Clínica de Fisioterapia do Centro Universitário de Lavras. O responsável pela clínica assinou um termo de autorização, e os voluntários assinaram um Termo de Consentimento Livre e Esclarecido, seguido pela aplicação de uma ficha de anamnese e Mini Exame do Estado Mental (MEEM). Posteriormente foram aplicados a Escala de Qualidade de Vida Específica para AVC (EQVE-AVC) e Índice de Barthel Modificado (IBM). Resultados. Houve uma correlação significativa entre a EQVE-AVC e IBM. Dentre os domínios avaliados pela EQVE-AVC, os itens: função do membro superior, trabalho/produtividade, cuidados pessoais, ânimo e relações familiares tiveram correlação significativa com a independência funcional. Conclusão. Este estudo evidenciou uma diminuição da QV e do grau de independência funcional dos pacientes com AVC. Conclui-se que há uma relação diretamente proporcional entre a QV e o grau de independência funcional dos voluntários avaliados.
\end{abstract}

Unitermos. Acidente vascular cerebral; Neurofuncional; Geriatria

\begin{abstract}
Objective. To correlate the quality of life (QOL) with the degree of functional independence of individuals que suffered cerebral vascular accident (CVA). Method. The sample of this study consisted of 12 patients of both genders, with a mean age of $60.58 \pm 10.34$ years, no regular exercise practitioners who have suffered stroke from 6 months to 9 years and were in physical therapy at University Center of the Physiotherapy Clinic of Lavras. The head of the clinic signed an authorization form, and volunteers signed a Consent Agreement and Informed, followed by application of a form of history and Mini Mental State Examination (MMSE). Later it was applied to Quality of Life Scale Specific for stroke (SS-QOL) and Modified Barthel Index (IBM). Results. There was a significant correlation between SS-QOL and IBM. Among the areas evaluated by SS-QOL, the items: upper limb function, work/productivity, personal care, courage and family relationships had significant correlation with functional independence. Conclusions. This study showed a decrease in QOL and the degree of operational independence of stroke patients. We conclude that there is a directly proportional relationship between $\mathrm{QOL}$ and the degree of functional independence of the assessed volunteers.
\end{abstract}

Keywords. Quality of life; Neurofunctional; Geriatrics 
Endereço para correspondência: Laiz Helena de Castro Toledo Guimarães. Centro Universitário de Lavras - UNILAVRAS - Departamento de Fisioterapia. Rua Padre José Poggel, 506, Centenário. CEP: 37200-000, Lavras - MG, Brasil. E-mail: laizunilavras@hotmail.com

\section{INTRODUÇÃO}

O Acidente Vascular Cerebral (AVC) é definido pela Organização Mundial da Saúde (OMS) como uma disfunção neurológica aguda de origem vascular, com sinais e sintomas que correspondem ao comprometimento de áreas focais do cérebro ${ }^{1}$. Ele é relatado como um déficit neurológico de início súbito ocasionado por uma alteração vascular que promove a interrupção do fluxo sanguíneo para uma área específica, que pode ser isquêmico ou hemorrágico².

No Brasil, o AVC é primeira causa de morte e incapacidade. Dados do estudo prospectivo nacional indicaram uma incidência anual de 108 casos por 100 mil habitantes ${ }^{3}$.

Em geral, o AVC acomete pessoas acima de 65 anos, e a incidência aumenta rapidamente com o aumento da idade; sendo que após os 55 anos o risco dobra a cada 10 $\operatorname{anos}^{4}$.

O conceito de Qualidade de Vida (QV) é multidimensional e refere-se à percepção subjetiva do indivíduo em relação à sua vida, relacionamento com a família e a sua própria saúde, questões financeiras, moradia, independência, vida social e atividades de lazer ${ }^{5}$. 
Os pacientes que sobrevivem ao AVC sofrem uma diminuição na sua QV como consequência das limitações e incapacidades física e cognitiva ${ }^{6}$.

As sequelas resultantes do AVC podem interferir no bem-estar físico, emocional, econômico e social, causando prejuízos na QV e na independência funcional dos indivíduos. A realização de uma avaliação para mensurar o impacto do AVC na QV e na independência funcional é de extrema importância para elaboração de programas de reabilitação que levem em consideração todos os fatores que estão envolvidos na QV desses indivíduos, porém, há uma escassez de estudos mensurando o quanto a QV e a independência funcional está prejudicada no pós-AVC; escassez de estudos que utilizem as mesmas escalas deste estudo; e até o momento não existem estudos avaliando esses aspectos nos pacientes em tratamento na Clínica de Fisioterapia Risoleta Neves. Portanto, tem-se a necessidade de avaliar e quantificar os prejuízos causados pelo AVC, para que intervenções possam ser realizadas.

Neste contexto, o objetivo do presente estudo foi correlacionar a QV com o grau de independência funcional de indivíduos que sofreram AVC.

\section{MÉTODO}

\section{Amostra}

Trata-se de um estudo observacional transversal, aprovado pelo Comitê de Ética em Pesquisa envolvendo 
Seres Humanos do Centro Universitário de Lavras, sob o protocolo CAAE 37456514.0.0000.5116, estando de acordo com a resolução 466/12 do Conselho Nacional de Saúde.

Foi desenvolvido com uma amostra composta por 12 indivíduos que sofreram AVC há pelo menos seis meses e que se encontravam em tratamento fisioterapêutico na Clínica de Fisioterapia Risoleta Neves - UNILAVRAS.

Adotou-se como critérios de inclusão: indivíduos de ambos os gêneros; com diagnóstico médico de AVC há pelo menos 6 (seis) meses, por já possuírem um estágio crônico em que as sequelas já estão instaladas; que não apresentavam alterações cognitivas, ou seja, pontuação $\geq 24$ pontos $^{7}$ no Mini Exame do Estado Mental (MEEM); indivíduos que apresentaram a fala preservada (item observado no momento de aplicação da ficha de anamnese). Critérios de exclusão: Indivíduos que praticassem exercício físico regularmente, pois o exercício físico melhora a QV pós-AVC ${ }^{9}$; indivíduos que não aceitassem participar da pesquisa e/ou aqueles que optassem por desistir da pesquisa a qualquer momento.

\section{Procedimento}

O estudo foi conduzido inicialmente com a assinatura do termo de autorização pelo responsável pela clínica. Em seguida, os voluntários assinaram o Termo de Consentimento Livre e Esclarecido, seguido pela aplicação de uma ficha de anamnese elaborada pelos autores para caracterização da amostra e MEEM para triagem dos 
indivíduos. Posteriormente foram aplicados a Escala de Qualidade de Vida Específica para o AVC (EQVE-AVC) ${ }^{8}$ e Índice de Barthel Modificado (IBM) ${ }^{9}$. O período de coleta de dados foi de novembro/2014 a junho/2015.

As avaliações foram realizadas individualmente em sala com privacidade no setor de Fisioterapia do Centro Universitário de Lavras, sempre após o horário do atendimento fisioterapêutico.

O MEEM é um instrumento amplamente utilizado, cujo objetivo é avaliar rapidamente as habilidades cognitivas e, assim, identificar possível comprometimento cognitivo. Avalia parâmetros quanto à orientação temporal e espacial, memória imediata, cálculo, linguagem e apraxia construtiva. Foi aplicada a versão brasileira do MEEM para identificar declínios cognitivos e selecionar os pacientes que estavam aptos a responder a EQVE-AVC ${ }^{10}$.

A EQVE-AVC é uma versão originada da Stroke Specific Quality of Life Scale, que foi desenvolvida na língua inglesa ${ }^{11}$, sendo o primeiro instrumento específico para avaliação da QV em indivíduos que sofreram AVC. Foi traduzida e adaptada transculturalmente para o português do Brasil $^{12}$ de acordo com regras padronizadas. A EQVEAVC é o único instrumento que avalia a QV em pacientes com AVC que apresenta tradução transcultural $e$ propriedades psicométricas avaliadas para uso no Brasil, além de apresentar boa confiabilidade, é uma ferramenta bastante útil e simples na sua aplicação. É composta por 49 itens subdivididos em 12 domínios (mobilidade, função do 
membro superior, trabalho/produtividade, visão, linguagem, cuidados pessoais, energia, ânimo, modo de pensar, comportamento, relações sociais e relações familiares). As possibilidades de respostas são dadas com relação à semana anterior e quantificadas de 1 a 5 , sendo o escore mínimo de 49 e máximo 245 pontos $^{8}$. Quanto maior a pontuação obtida, melhor a QV. Escores inferiores a 147 pontos são definidos como baixa $\mathrm{QV}^{13}$.

A primeira versão do índice de Barthel foi desenvolvida para mensurar o grau de dependência de indivíduos com doenças crônicas após um período de reabilitação ${ }^{14}$. A segunda versão, IBM, procura estabelecer o grau de dependência do sujeito em dez categorias funcionais: higiene pessoal, banho, alimentação, toalete, subir escadas, vestuário, controle vesical, controle intestinal, deambulação e transferências. Para cada categoria é dada uma pontuação de acordo com o nível de dependência, sendo 0 incapaz de realizar a tarefa e até 15 quando se apresenta totalmente independente. O grau de dependência foi estabelecido de acordo com o escore total alcançado: total (0-24), grave (25-50), moderado (51-75), mínimo (76-99) e independente $(100)^{9}$.

\section{Análise Estatística}

A análise estatística das medidas descritivas foi realizada através da média e desvio padrão; e para correlacionar os dados obtidos entre a EQVE-AVC e IBM foi utilizado o teste de correlação de Spearman. 


\section{RESULTADOS}

A amostra deste estudo foi composta por 12 indivíduos de ambos os gêneros, não praticantes de exercício físico regular, que sofreram AVC no período de 6 meses a 9 anos, e quantidade máxima de 3 AVCs. A média de idade dos

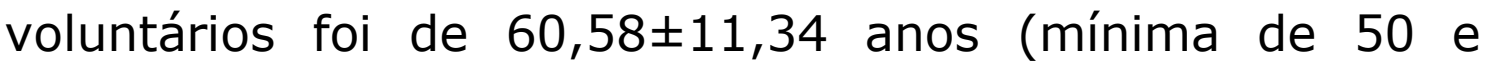
máxima 78 anos).

A partir da EQVE-AVC, foram extraídos dados acerca de como estava a QV dos pacientes em relação à semana anterior ao dia da avaliação. A Figura 1 representa os domínios mais afetados após o AVC: andar (75\% relataram que está muito pior); fazer coisas para amigos (66,7\% relataram que está muito pior); uso de braços ou mãos (58,3\% relataram que está muito pior) e ânimo (58,3\% relataram que está muito pior).

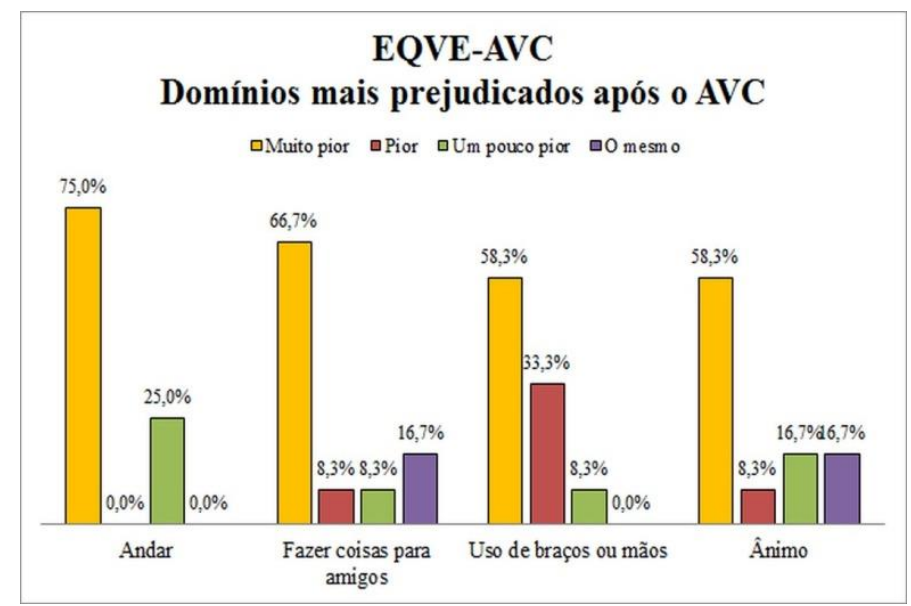

Figura 1. Domínios avaliados de acordo com a EQVE-AVC: andar, fazer coisas para amigos, uso de braços ou mãos, ânimo. 
O último domínio da EQVE-AVC se refere a seguinte pergunta: "No geral, minha qualidade de vida está?" Do total de respostas, $41,7 \%$ relataram estar muito pior, $16,7 \%$ pior, $16,7 \%$ um pouco pior, e $25 \%$ relataram estar o mesmo que antes do AVC ter ocorrido (Figura 2).

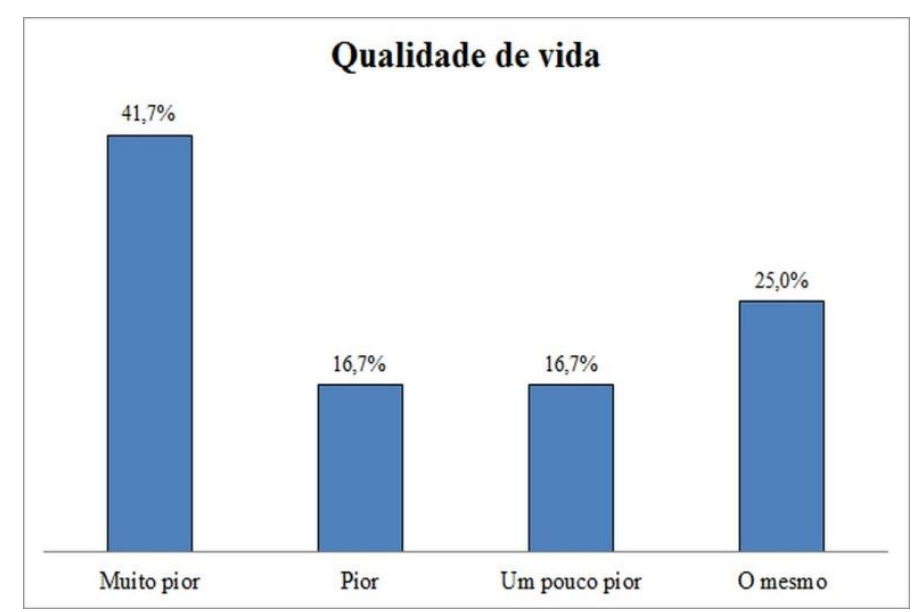

Figura 2. Representa como a QV dos indivíduos se encontra atualmente, comparando como estava antes do AVC, com opções de respostas: muito pior, pior, um pouco pior ou o mesmo.

De acordo com o IBM, os pacientes responderam perguntas sobre os domínios: higiene pessoal, banho, alimentação, toalete, subir escada, vestuário, controle esfincteriano (bexiga e intestino), deambulação ou cadeira de rodas e transferências cadeira/cama. A Figura 3 representa em porcentagem os graus de independência funcional dos pacientes avaliados, sendo: dependência total, grave, moderada, mínima ou independência.

A Tabela 1 apresenta as medidas descritivas (média e desvio padrão) e coeficiente de correlação de Spearman entre a QV e a independência funcional. 


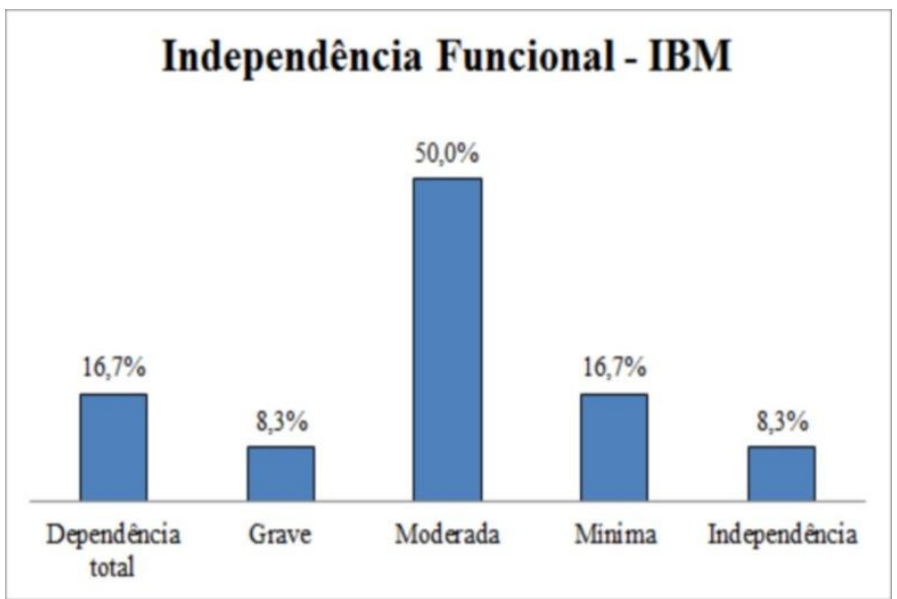

Figura 3. Representa como está a independência dos pacientes de acordo com o IBM.

Tabela 1. Medidas descritivas (média e desvio padrão) e coeficiente de correlação de Spearman entre a Qualidade de Vida e a Independência Funcional.

\begin{tabular}{cccc}
\hline $\begin{array}{c}\text { Instrumentos de } \\
\text { Avaliação }\end{array}$ & Média & Desvio Padrão & $\begin{array}{c}\text { Coeficiente de } \\
\text { Correlação }\end{array}$ \\
\hline EQVE-AVC & 157,75 & 33,91 & $0,732 * *$ \\
IBM & 62,42 & 23,52 &
\end{tabular}

** Correlação significante ao nível de $1 \%$.

A Tabela 2 apresenta as medidas descritivas (média e desvio padrão) e coeficiente de correlação de Spearman entre cada bloco do questionário EQVE-AVC e IBM. Os itens: função do membro superior e trabalho/produtividade tiveram correlação positiva entre a EQVEAVC e IBM ao nível de significância de 1\%. Já os itens: cuidados pessoais, ânimo e relações familiares tiveram correlação significativa entre a EQVE-AVC e IBM ao nível de 5\%. Os demais dados não obtiveram correlação positiva. 
Tabela 2. Medidas descritivas (média e desvio padrão) e coeficiente de correlação de Spearman entre cada bloco da EQVE-AVC e a independência funcional.

\begin{tabular}{cccc}
\hline Blocos & Média & Desvio Padrão & $\begin{array}{c}\text { Coeficiente de } \\
\text { Correlação }\end{array}$ \\
\hline Mobilidade & 19,00 & 8,88 & 0,512 \\
Função do Membro Superior & 17,00 & 4,13 & $0,787 * *$ \\
Trabalho/Produtividade & 8,00 & 3,13 & $0,833^{* *}$ \\
Visão & 14,67 & 2,99 & 0,439 \\
Linguagem & 20,33 & 4,14 & 0,276 \\
Cuidados pessoais & 17,50 & 5,70 & $0,684^{*}$ \\
Energia & 8,92 & 4,29 & $-0,421$ \\
Ânimo & 14,42 & 6,05 & $0,605^{*}$ \\
Modo de pensar & 9,08 & 3,63 & $-0,237$ \\
Comportamento & 10,00 & 4,75 & $-0,061$ \\
Relações Sociais & 11,25 & 6,65 & 0,170 \\
Relações Familiares & 7,58 & 4,50 & $0,691 *$ \\
\hline
\end{tabular}

* Correlação significante a 5\%. ** Correlação significante ao nível de $1 \%$.

\section{DISCUSSÃO}

A avaliação da QV em pacientes que sofreram AVC vem sendo crescente na área da saúde, à medida que a sobrevida aumenta, aumenta a necessidade de conhecimento na área acerca das sequelas.

A média de idade de 60,58 anos encontrada nos voluntários deste estudo foi estabelecida de acordo com a demanda de pacientes que se encontravam em tratamento. Houve a tentativa de estabelecer valores entre 55 e 65 anos, porém, se os pacientes com 50 anos fossem excluídos diminuiria o tamanho da amostra. Esse achado se assemelha a outros estudos já realizados ${ }^{15-18}$, em que a média de idade era superior a 60 anos. Este fato se deve tanto a uma maior expectativa de vida da população, quanto ao aumento da sobrevida nos pacientes com AVC e à melhoria dos atendimentos ${ }^{19}$.

É de fundamental importância a realização de atividades de reabilitação no pós-AVC. No presente estudo, 
todos os voluntários realizavam fisioterapia duas vezes por semana, o que garante sucesso no tratamento, e já se encontravam em tratamento fisioterapêutico no período mínimo de 2 meses e máximo de 3 anos. Há um estudo que afirma que os pacientes pós-AVC quando enquadrados em um programa de fisioterapia tem ganhos funcionais nas atividades de vida diária ${ }^{20}$.

A QV tende a ficar comprometida quando o indivíduo é afetado por doenças crônicas e quando as sequelas já estão instaladas $^{13}$. No presente estudo, os domínios mais prejudicados após o AVC foram: andar (75\% relataram que está muito pior), fazer coisas para amigos $(66,7 \%$ relataram que está muito pior); uso de braços ou mãos e ânimo (58,3\% relataram que está muito pior). Após a ocorrência do AVC se instala um quadro agudo, porém, é uma doença crônica em que a partir dos 6 meses as sequelas já podem estar instaladas. Esta afirmação esclarece porque a amostra foi selecionada com pacientes que sofreram AVC há pelo menos 6 meses.

Nos pacientes do estudo, o ato de andar está muito pior após o AVC, o que está de acordo com um estudo já realizado $^{21}$, em que maioria dos pacientes que sofreram AVC (75\%) consegue andar, sendo que nem sempre este retorno é funcionalmente satisfatório. Os pacientes pósAVC tem dificuldade para manter o peso no hemicorpo afetado, interferindo no controle postural e gerando dificuldades para realizar movimentos de tronco e membros ${ }^{22}$. Segundo estudo já realizado, o maior problema 
encontrado nos pacientes com comprometimento nervoso e musculoesquelético é a perda da habilidade de andar ${ }^{23}$.

Os itens: função do membro superior e trabalho/produtividade tiveram correlação significativa ao nível de $1 \%$ com a independência funcional, portanto, à medida que essas variáveis aumentam, melhora a QV, pois a independência funcional também aumenta. Após o AVC os indivíduos podem desenvolver estratégias compensatórias para utilizar o membro superior ${ }^{24}$. 58,3\% dos pacientes relataram que o uso de braços ou mãos está muito pior que antes; e este fato pode estar relacionado com o não aprendizado sobre esse mecanismo compensatório. $\mathrm{Na}$ tentativa de se manter independente, o paciente tenta realizar as atividades dentro do possível, sendo muitas vezes movimentos incoordenados, ou não sabendo distribuir a força da maneira correta, com movimentos estereotipados.

Quando se comparou como se encontrava a QV dos indivíduos avaliados na semana anterior à avaliação, foi possível observar que $41,7 \%$ dos indivíduos relataram que a QV está muito pior do que antes do AVC ter ocorrido. Apenas 25\% relataram que está a mesma coisa; e uma pequena parcela relatou que está pior $(16,7 \%)$ ou um pouco pior que antes $(16,7 \%)$. Autores concordam que 0 AVC é uma das doenças mais incapacitantes, influenciando desfavoravelmente a QV do paciente ${ }^{25}$.

Em relação à independência funcional avaliada através do IBM, foi observado que $16,7 \%$ dos pacientes avaliados 
apresentaram dependência total, 8,3\% dependência grave, $50 \%$ moderada, $16,7 \%$ dependência mínima e 8,3\% independentes. Esses dados estão de acordo com outro estudo já realizado ${ }^{26}$, em que foi avaliado o grau de dependência dos pacientes que sofreram AVC, e os achados foram: $19,2 \%$ independentes; $57,7 \%$ dependência ligeira a moderada; $11,6 \%$ apresentam uma dependência grave e $11,5 \%$ totalmente dependentes.

Os itens relacionados aos cuidados pessoais, ânimo e relações familiares obtiveram correlação significativa, ou seja, esses domínios influenciam diretamente na independência funcional. Portanto, quanto maior for a capacidade de cuidados pessoais, animação e boas relações familiares, maior será a independência funcional destes indivíduos. Estudos mostraram que a assistência familiar quando positiva e de qualidade, melhora a $\mathrm{QV}$ dos pacientes pós-AVC ${ }^{27,28}$.

Os domínios: mobilidade, energia, modo de pensar, comportamento e relações sociais, não tiveram correlação significativa com a independência funcional. Estes aspectos discordam dos achados de outros autores ${ }^{29}$, que demonstraram forte correlação nesses domínios em seu estudo com pacientes que sofreram AVC, porém, esses domínios foram correlacionados somente com a QV, e não com independência funcional.

Segundo dados do presente estudo, que foi utilizado a EQVE-AVC e IBM, é possível afirmar que houve uma correlação positiva e significativa entre a QV e a 
independência funcional. Pode-se concluir que, neste estudo, à medida que a independência funcional aumenta, a QV também aumenta, sendo variáveis diretamente proporcionais.

\section{CONCLUSÃO}

Os achados evidenciaram uma diminuição da QV e do grau de independência funcional em pacientes com AVC que estavam em tratamento fisioterapêutico na Clínica de Fisioterapia Risoleta Neves. Há uma relação diretamente proporcional entre a QV e o grau de independência funcional dos voluntários avaliados.

Uma limitação do estudo foi o tamanho reduzido da amostra, portanto, sugere-se que novos trabalhos sejam realizados com número maior de indivíduos, pois é um assunto de grande importância na área da fisioterapia.

Como os resultados obtidos com o presente estudo apontaram uma diminuição da QV e da independência funcional dos indivíduos avaliados, teve-se a necessidade de realizar uma palestra para expor os resultados obtidos com este estudo e conscientizar os pacientes acerca do que pode ser feito na tentativa de minimizar os prejuízos causados pelo AVC, incentivando a movimentação no hemicorpo afetado e instruindo o paciente quanto à inclusão de outras áreas que não somente a fisioterapia. 


\section{AGRADECIMENTOS}

Os autores agradecem à FAPEMIG (Fundação de Amparo à Pesquisa do Estado de Minas Gerais) pelo suporte financeiro e aos responsáveis pela Clínica de Fisioterapia Risoleta Neves por permitirem a realização deste estudo.

\section{REFERÊNCIAS}

1.Gillen G. Acidente vascular cerebral. In: Pedretti LW, Early MB. Terapia ocupacional: capacidades práticas para as disfunções físicas. São Paulo: Roca, 2005, p.675-703.

2.Faria I. Neurologia adulto. In: Cavalcanti A, Galvão C. Terapia ocupacional: fundamentação \& prática. Rio de Janeiro: Guanabara Koogan, 2007, p.187-90.

3.BRASIL. Ministério da Saúde. Secretaria de Atenção à Saúde. Departamento de Ações Programáticas Estratégicas. Diretrizes de atenção à reabilitação da pessoa com acidente vascular cerebral/Ministério da Saúde, Secretaria de Atenção à Saúde, Departamento de Ações Programáticas Estratégicas. Brasília: Ministério da Saúde, 2013.

4.Seidl EMF, Zannon CMLC. Qualidade de vida e saúde: aspectos conceituais e metodológicos. Cad Saúde Pública 2004;20:580-8. http://dx.doi.org/10.1590/S0102-311X2004000200027

5.Costa IMPDEF. A Qualidade de vida de pacientes sobreviventes de acidente vascular encefálico (Dissertação). Aracajú: Universidade Tiradentes, 2008, 67f.

6. Murden RA, McRae TD, Kaner S, Bucknam ME. Mini-Mental State Exam scores vary with education in blacks and whites. J Am Geriatr Soc. $1991 ; 39: 149-155$.

7.Billinger SA, Arena R, Bernhardt J, et al. Physical activity and exercise recommendations for stroke survivors: a statement for healthcare professionals from the American Heart Association/American Stroke Association. Stroke 2014;45:2532-53 http://dx.doi.org/10.1161/STR.0000000000000022

8.Santos AS. Validação da escala de avaliação da qualidade de vida na doença cerebrovascular isquêmica para a língua portuguesa (Tese). São Paulo: Faculdade de Medicina, Universidade de São Paulo, 2007, 126f.

9.Shah S, Vanclay F, Cooper B. Improving the sensitivity of the Barthel Index for stroke rehabilitation. J Clin Epidemiol 1989;42:7039.

10.Scalzo PL, Souza ES, Moreira AGO, Vieira DAF. Qualidade de vida em pacientes com Acidente Vascular Cerebral: clínica de fisioterapia Puc Minas. Betim. Rev Neurocienc 2010;18:139-44. 
11.Williams LS, Weinberger M, Harris LE, Clark DO, Biller J. Development of a stroke-specific quality of life scale. Stroke 1999;30:1362-9.

12.Santos AS. Questionário específico de avaliação da qualidade de vida em pacientes portadores de doença cérebro vascular do tipo isquêmica: tradução e adaptação cultural para a língua portuguesa falada no Brasil (Tese). Santa Catarina: Universidade Federal de Santa Catarina, Florianópolis, 2000, $108 \mathrm{f}$.

13.Rangel ESS, Belasco AGS, Diccini S. Qualidade de Vida de Pacientes com Acidente vascular cerebral em reabilitação. Acta Paul Enferm 2013;26:205-12. http://dx.doi.org/10.1590/S010321002013000200016

14. Hsueh IP, Lin JH, Jeng JS, Hsieh CL. Comparison of the psychometric characteristics of the functional independence measure, 5 item Barthel index, and 10 item Barthel index in patients with stroke. J Neurol Neurosurg Psychiatr 2002;73:188-90.

15. Benvegnu AB, Gomes LA, Souza CT, Cuadros TBB, Pavão LW, Ávila SN. Avaliação da medida de independência funcional de indivíduos com sequelas de acidente vascular encefálico (AVE). Rev Ciência Saúde 2008;1:71-7.

16.Caneda MAG, Fernandes JG, Almeida AG, Mugnol FE. Confiabilidade de Escalas de Comprometimento Neurológico em Pacientes com Acidente Vascular Cerebral. Arq Neuropsiquiatr 2006;64:690-7. http://dx.doi.org/10.1590/S0004282X2006000400034

17.Delboni MCC, Malengo PCM, Schmidt EPR. Relação entre os aspectos das alterações funcionais e seu impacto na qualidade de vida das pessoas com sequelas de Acidente Vascular Encefálico (AVE). O Mundo da Saúde 2010;34:165-75.

18.Tsukamoto HF, Picinatto, $A E$, Cavalini CA, Bortolloti LF. Análise da independência funcional, qualidade de vida, força muscular respiratória e mobilidade torácica em pacientes hemiparéticos submetidos a um programa de reabilitação: estudos de caso. Semina: Ciências Biológicas da Saúde 2010;31:63-9. http://dx.doi.org/10.5433/1679-0367.2010v31n1p63

19.Pereira ABCNG, Alvarenga $H$, Júnior RSP, Barbosa MTS. Prevalência de acidente vascular cerebral em idosos no Município de Vassouras, Rio de Janeiro, Brasil, através do rastreamento de dados do Programa Saúde da Família. Cad Saúde Pública 2009;25:1929-36. http://dx.doi.org/10.1590/S0102-311X2009000900007

20.Rodrigues JE, SÁ MS, Alouche SR. Perfil dos pacientes acometidos por AVE tratados na clínica escola de fisioterapia da UMESP. Rev Neurocienc 2004;12:117-22.

21.Ottoboni C, Fontes SV, Fukujima MM. Estudo Comparativo entre a Marcha Normal e a de Pacientes Hemiparéticos por Acidente Vascular Encefálico: Aspectos Biomecânicos. Rev Neurocienc 2002;10:10-6.

22. Chagas EF, Tavares MCGCF. Simetria e transferência de peso do hemiplégico: relação dessa condição com o desempenho de suas 
atividades funcionais. Rev Fisioter USP 2001;8:40-50. https://doi.org/10.1590/fpusp.v8i1.79397

23.Alonso VK, Okaji SS, Pinheiro MT, Ribeiro CM, Souza HP, Santos SS, et al. Análise Cinemática da Marcha em Pacientes Hemiparéticos. FisioBrasil 2002;1:16-21.

24.Faria I. Função do membro superior em hemiparéticos crônicos: análise através da classificação internacional de funcionalidade, incapacidade e saúde (Dissertação). Belo Horizonte: Universidade Federal de Minas Gerais, 2008, $114 \mathrm{f}$.

25. Oliveira MR, Orsini M. Escalas de avaliação da qualidade de vida em pacientes brasileiros após acidente vascular encefálico. Rev Neurocienc 2009;17:255-62.

26. Azeredo Z, Matos E. Grau de Dependência em Doentes que sofreram AVC. RFML 2003;8:199-204.

27. Lima ML. Qualidade de vida de indivíduos com acidente vascular encefálico e de seus cuidadores (Dissertação). Ribeirão Preto: Universidade de São Paulo, Faculdade de Medicina, 2010, $124 \mathrm{f}$.

28.Lee ACK, Tang SW, Tsoi TH, Fong DYT, Yu GKK. Predictors of poststroke quality of life in older Chinese adults. J Adv Nurs 2008;65:554-64. https://doi.org/10.1111/j.1365-2648.2008.04918.x 29.Gunaydin R, Karatepe AG, Kaya T, Ulutas O. Determinants of quality of life (QoL) in elderly stroke patients: a short-term follow-up study. Arch Gerontol

Geriatr 2011;53:19-23. https://doi.org/10.1016/j.archger.2010.06.004 